\title{
Hydrogen Molecules in Crystalline Silicon Treated with Atomic Hydrogen
}

\author{
K. Murakami, ${ }^{1}$ N. Fukata, ${ }^{1}$ S. Sasaki, ${ }^{1}$ K. Ishioka, ${ }^{2}$ M. Kitajima, ${ }^{2}$ S. Fujimura, ${ }^{3}$ J. Kikuchi, ${ }^{3}$ and H. Haneda ${ }^{4}$ \\ ${ }^{1}$ Institute of Materials Science, University of Tsukuba, Tennoudai 1-1-1, Tsukuba, Ibaraki 305, Japan \\ ${ }^{2}$ National Research Institute for Metals, Sengen 1-2-1, Tsukuba, Ibaraki 305, Japan \\ ${ }^{3}$ Process Development Division, Fujitsu Ltd., Kawasaki, Kanagawa 211, Japan \\ ${ }^{4}$ National Institute for Research in Inorganic Materials, Namiki 1-1, Tsukuba, Ibaraki 305, Japan
}

(Received 4 March 1996; revised manuscript received 17 May 1996)

\begin{abstract}
We report the first observation of the vibrational Raman spectrum of hydrogen molecules $\mathrm{H}_{2}$ in crystalline silicon treated with hydrogen atoms at $400{ }^{\circ} \mathrm{C}$. The Raman spectrum of $\mathrm{H}_{2}$ in silicon observed at room temperature exhibits a frequency shift of around $4158 \mathrm{~cm}^{-1}$ and a very broad half-width of approximately $34 \mathrm{~cm}^{-1}$. An isotope shift was also detected at around $2990 \mathrm{~cm}^{-1}$ in silicon treated with deuterium atoms at $400{ }^{\circ} \mathrm{C}$. The frequency shifts of the observed lines are in close agreement with those reported for $\mathrm{H}_{2}$ and $\mathrm{D}_{2}$ in the gas, liquid, and solid phases. [S0031-9007(96)01349-X]
\end{abstract}

PACS numbers: 61.72.Ji, 76.30.-v, 78.30.-j

Hydrogen is an important impurity in silicon, and is incorporated during the various stages of semiconductor processing. In crystalline silicon treated with hydrogen atoms, an electron spin resonance spectrum of isolated hydrogen has only been detected for silicon implanted at low temperatures with protons [1]. Thus, isolated atomic hydrogen is not stable in crystalline silicon and forms complexes with other defects or forms hydrogen clusters that have not been unambiguously observed previously. Recent calculations predict that in silicon, among the various forms of hydrogen-related defects, a hydrogen molecule oriented along either the $\langle 111\rangle$ or $\langle 100\rangle$ axis at the tetrahedral interstitial site has a relatively low energy [2,3]. However, no direct observation of hydrogen molecules has been reported so far [1], although the importance of interstitial $\mathrm{H}_{2}$ molecules has been suggested indirectly by a few experimental studies [4]. An electron paramagnetic resonance spectrum for a hydrogen molecule in silicon damaged with $\mathrm{H}^{+}$ion implantation has been reported recently [5]; however, no isotope effects have been reported, and these results have been controversial [6,7]. A metastable configuration for another type of hydrogen molecule has also been proposed [7]. This consists of a bond-center (BC) hydrogen and a hydrogen at a neighboring tetrahedral interstitial site, and has an energy that is $0.01 \mathrm{eV}$ higher than the most stable hydrogen molecule. Recently this metastable hydrogen molecule was reported to be observed also in $\mathrm{H}^{+}$implanted silicon [8]. For amorphous $\mathrm{Si}: \mathrm{H}$, on the other hand, hydrogen molecules in voids have been directly detected by a few measurements such as nuclear spin resonance [9] and infrared absorption [10]. The existence of deuterium molecules was also suggested by Raman measurement of the surface layer of amorphized graphite exposed to deuterium glow discharge [11]. These molecular states in amorphous solids are inferred to be entirely different from those of a hydrogen molecule in crystalline silicon which are predicted to be located at the tetrahedral interstitial sites. Thus it is of great importance and of interest to find the condition for realizing the formation of hydrogen molecules in undamaged, crystalline silicon and to investigate their properties if possible.

In this Letter, we report direct spectroscopic evidence for the existence of hydrogen molecules in crystalline silicon through their Raman spectrum and the effect of the replacement of the hydrogen atoms by deuterium. In particular, the isotope shift of the Raman lines is important for the identification of hydrogen molecules, since it gives a direct measure of the hydrogen-related defects and is very sensitive to the structures. The values of the observed Raman shifts are well consistent with those reported for $\mathrm{H}_{2}$ and $\mathrm{D}_{2}$ in the gas, liquid, and solid states, while the halfwidths of the lines are 2 orders of magnitude broader than those reported in the literature $[12,13]$.

Floating-zone (FZ), $p$-type silicon wafers that had been implanted with phosphorus at $50 \mathrm{keV}$ with doses of $(1-5) \times 10^{14} / \mathrm{cm}^{2}$ and thermally annealed at $900{ }^{\circ} \mathrm{C}$ for $30 \mathrm{~min}$ were used for atomic-hydrogen introduction. Hydrogenation was performed at various substrate temperatures ranging from 60 to $400{ }^{\circ} \mathrm{C}$ for $3 \mathrm{~h}$ by the remote treatment with downstream hydrogen atoms from hydrogen plasmas which were generated by microwave irradiation in a microwave cavity $[14,15]$. Both surfaces of the wafers were mirror polished in order to accurately measure and control the temperatures during atomic-hydrogen treatment by means of an infrared-laser interference. A FZ, $p$-type sample without $\mathrm{P}^{+}$implantation was treated under similar conditions for comparison. Deuterium treatment was also done in the same way as hydrogenation. Details of the hydrogen atom treatment were reported in previous papers $[12,13]$.

Electron spin resonance (ESR) measurements were done at lower temperatures of 4.2 and $110 \mathrm{~K}$ with an $X$-band spectrometer. Raman scattering measurements were performed at room temperature in a $90^{\circ}$-scattering configuration with the $514.5 \mathrm{~nm}$ line of a cw argon-ion 
laser as the Raman excitation light. The scattered light was collected with a lens, dispersed with a triple grating monochromator (JASCO TRS-600) with a wave-number resolution of $0.2-0.3 \mathrm{~cm}^{-1}$ and detected with a spectrometric multichannel analyzer (Princeton Instruments Inc. D/SIDA700). The measured range of frequency shift was from 300 to $4210 \mathrm{~cm}^{-1}$. The depth profiles of hydrogen and deuterium were obtained from secondary ion mass spectrometry (SIMS) measurements which were carried out with an $\mathrm{O}^{2+}$ or $\mathrm{Cs}^{+}$ion beam with a CAMECA IMS 4f system.

ESR spectra were measured for $\mathrm{P}$ donors and/or conduction electrons in silicon samples treated at various substrate temperatures. It has been found previously by ESR that the most effective substrate temperature for the hydrogen passivation of $\mathrm{P}$ donors ranges from 100 to $120^{\circ} \mathrm{C}$ and that $\{111\}$ hydrogen-related platelets [16] are effectively formed at substrate temperatures between 150 and $250{ }^{\circ} \mathrm{C}$ [15]. Figure 1 shows typical results for the ESR linewidth and the Raman intensity of the optical phonon at $521 \mathrm{~cm}^{-1}$ for a silicon sample with $5 \times 10^{14} \mathrm{P}^{+} / \mathrm{cm}^{2}$ as a function of the substrate used for the hydrogen-atom treatment. The broadening of the ESR linewidth seen in the temperature range of 150 to $250^{\circ} \mathrm{C}$ is mainly caused by the hyperfine interaction between high density of hydrogen atoms (nuclear spin 1/2) in the platelets and the donor or conduction electrons $[15,17]$. As the substrate temperature is increased above $250^{\circ} \mathrm{C}$, the formation of platelets is suppressed and other hydrogen-related defects are produced. This corresponds to the decrease in the ESR linewidth

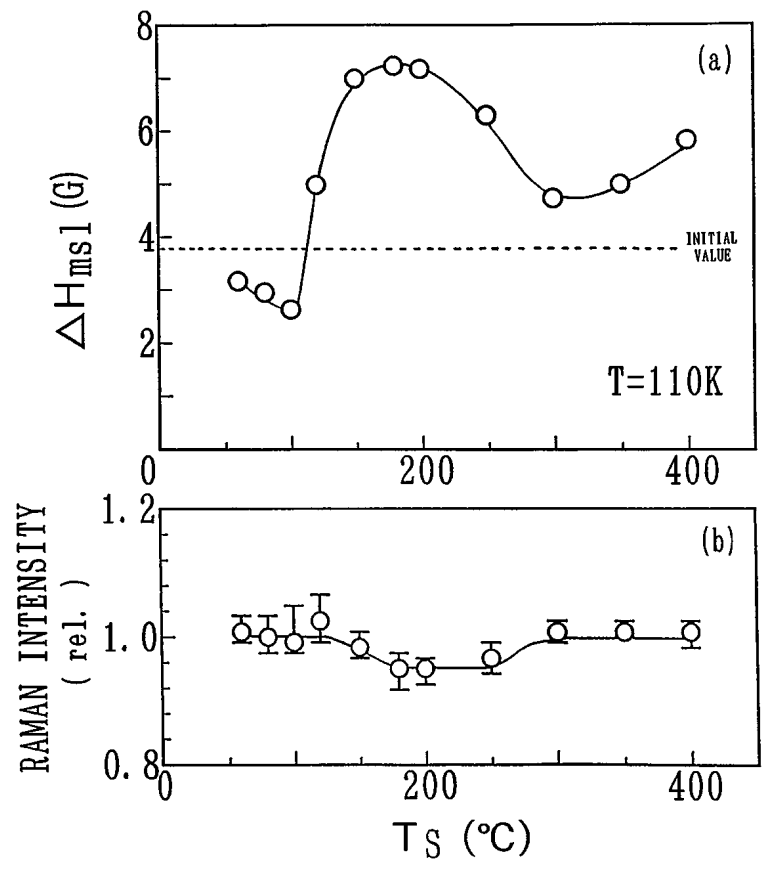

FIG. 1. (a) ESR linewidth of the conduction electrons measured at $110 \mathrm{~K}$ and (b) relative Raman intensity of the optical phonon $\left(521 \mathrm{~cm}^{-1}\right)$ as a function of the substrate temperature $T_{s}$ during hydrogen-atom treatment. The initial ESR linewidth observed before hydrogen-atom treatment is indicated by the dotted line. seen from 250 to $300{ }^{\circ} \mathrm{C}$ and to a slight increase above $300{ }^{\circ} \mathrm{C}$. The amount of incorporated hydrogen increases with increasing substrate temperature above $300^{\circ} \mathrm{C}$, as will be shown below with SIMS measurement. This suggests that some new hydrogen-related defects with no significant hyperfine interaction are produced. It is likely that one candidate is the hydrogen molecule $\mathrm{H}_{2}$, as suggested by Stutzmann et al. [4], since para- $\mathrm{H}_{2}$ has a total nuclear spin $I$ of 0 and has a lower energy than ortho- $\mathrm{H}_{2}$ with a nuclear spin of 1 . In order to look for hydrogen molecules in silicon samples treated above $300{ }^{\circ} \mathrm{C}$, we measured Raman spectra for several samples and also investigated the isotopic substitution, $\mathrm{D}$ for $\mathrm{H}$. The intensity of the Raman line of the longitudinal-optical (LO) phonon at $521 \mathrm{~cm}^{-1}$ is found to decrease slightly for atom treatment temperatures between 150 and $250{ }^{\circ} \mathrm{C}$, as shown in Fig. 1(b). This indicates that the silicon crystallinity degrades at these temperatures, presumably due to the growth of platelets.

Figure 2 shows typical Raman spectra obtained for four kinds of samples without $\mathrm{P}$ implantation and with a dose of $5 \times 10^{14} \mathrm{P}^{+} / \mathrm{cm}^{2}$; i.e., silicon samples treated at $400{ }^{\circ} \mathrm{C}$ with hydrogen atom (HAT $-400{ }^{\circ} \mathrm{C}$ ), treated at $400{ }^{\circ} \mathrm{C}$ with deuterium atom $\left(\mathrm{DAT}-400^{\circ} \mathrm{C}\right.$ ), and treated at $180{ }^{\circ} \mathrm{C}$ with hydrogen atom (HAT- $180^{\circ} \mathrm{C}$ ). We observed that there are no significant differences in the phonon correlation length [18] between two samples without hydrogen atom treatment for unimplanted silicon and P-implanted silicon followed by annealing at $900{ }^{\circ} \mathrm{C}$. A broad Raman line near $4158 \mathrm{~cm}^{-1}$ is seen clearly for both the samples of HAT $-400{ }^{\circ} \mathrm{C}$, with and without $\mathrm{P}$ ion implantation. We assign this vibrational line to the hydrogen molecule in crystalline silicon based upon its frequency which is close to those reported for hydrogen molecules in gas [12], liquid, and solid phases [13]. The half-width of the Raman line is 2 orders of magnitude broader than for the corresponding lines reported for liquid and solid $\mathrm{H}_{2}$ at lower temperatures [13]. Additionally, the Raman line at $4158 \mathrm{~cm}^{-1}$ is not seen for the sample HAT- $180{ }^{\circ} \mathrm{C}$ which exhibits broadening of the ESR linewidth, suggesting the existence of the platelets [15]. However, a broad Raman line can be seen instead at around $2140 \mathrm{~cm}^{-1}$ for sample HAT $-180{ }^{\circ} \mathrm{C}$. This Raman shift is similar to one previously assigned to Si-H vibrations associated with platelets [16]. A Raman line associated with $\mathrm{Si}-\mathrm{H}$ bond vibration is also observed at $2095 \mathrm{~cm}^{-1}$ for the sample HAT $-400{ }^{\circ} \mathrm{C}$. The shift to a lower wave number compared to that of the HAT- $180{ }^{\circ} \mathrm{C}$ sample means that the $\mathrm{Si}-\mathrm{H}$ bond is changed to a more relaxed state than for the $\mathrm{Si}-\mathrm{H}$ bond with strong compression in the platelets. For a sample treated with atomic hydrogen at $60{ }^{\circ} \mathrm{C}$, no Raman lines were observed near 4158, 2140, or $2095 \mathrm{~cm}^{-1}$. This indicates that hydrogen-related defects, such as the P-H complex $[1,13]$, other than hydrogen molecules and platelets are formed for an atom treatment temperature of $60{ }^{\circ} \mathrm{C}$.

It should also be stressed that the deuterium isotope effect can be clearly observed for the sample DAT- $400{ }^{\circ} \mathrm{C}$ in Fig. 2; i.e., at around $2990 \mathrm{~cm}^{-1}$, we see a broad Raman 


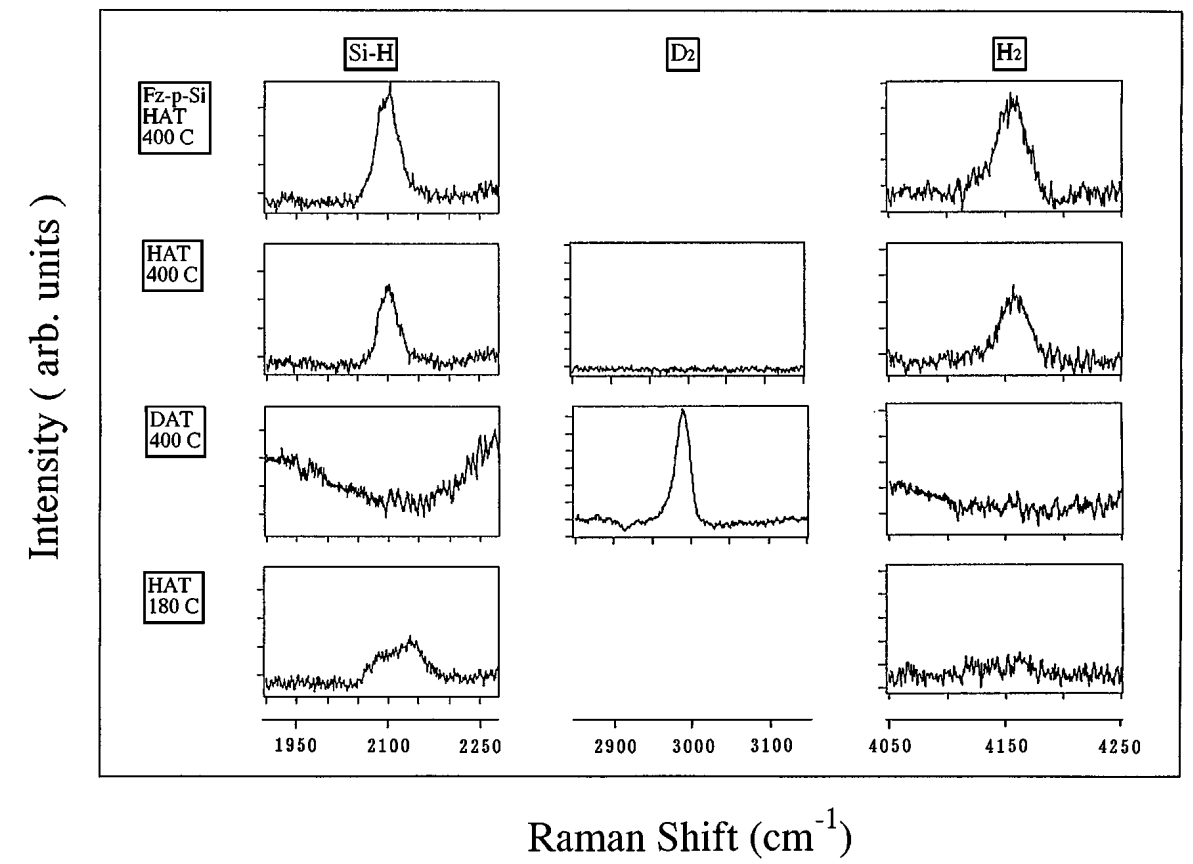

FIG. 2. Raman scattering spectra observed at three special ranges of the frequency shift for four kinds of samples. HAT and DAT mean "hydrogen-atom treatment" and "deuterium-atom treatment," respectively. The top is for the unimplanted, FZ silicon sample, and the others are for the implanted and subsequently annealed silicon samples.

line associated with deuterium molecule. The observed isotope shift is in excellent agreement with those reported for hydrogen and deuterium molecules, as shown in Table I. Thus, these results provide strong evidence for the existence of hydrogen molecules in the silicon treated with atomic hydrogen at $400{ }^{\circ} \mathrm{C}$.

The depth profiles of hydrogen and deuterium were obtained by SIMS measurements. Typical results for deuterium are shown in Fig. 3. The silicon sample treated at $180^{\circ} \mathrm{C}$ exhibits a peak with a high concentration of approximately $4 \times 10^{20} / \mathrm{cm}^{3}$ at around $400 \AA$. This presumably comes from deuterium atoms in $\{111\}$ platelets. On the other hand, the silicon samples treated at 300 and $400{ }^{\circ} \mathrm{C}$ exhibit a lower concentration, indicating deu- terium diffusion deeper into the silicon, and a peak in the deuterium concentration at around $750 \AA$. The maximum concentration for silicon treated at $400{ }^{\circ} \mathrm{C}$ is higher than that for silicon treated at $300{ }^{\circ} \mathrm{C}$. These concentrations are greater than those of the implanted phosphorus atoms. The hydrogen profiles showed similar results.

We have considered the possibility that the origin for the Raman line at $4158 \mathrm{~cm}^{-1}$ might be due to extended H-related defects such as hydrogen molecules and larger $\mathrm{H}$ clusters in a very thin surface layer with a thickness less than $100 \AA$ which might have been damaged, even with the remote plasma. In order to rule out this possibility, we etched off a surface layer by sequential rinses in $\mathrm{HNO}_{3}$ followed by HF and then measured the Raman spectrum.

TABLE I. $Q_{\Delta v=1}(J)$ vibrational $(v: 0-1)$ Raman lines. $v$ and $J$ are the vibrational quantum number and the rotational quantum number, respectively.

\begin{tabular}{|c|c|c|c|c|c|}
\hline & & $\begin{array}{l}\text { Observed Raman shifts } \\
\text { (half-widths) } \\
\left(\mathrm{cm}^{-1}\right)\end{array}$ & Solid $^{\mathrm{a}}$ & Liquid $^{\text {a }}$ & $\mathrm{Gas}^{\mathrm{b}}$ \\
\hline \multirow[b]{2}{*}{$\mathrm{H}_{2}$} & $\begin{array}{c}\text { para- } \mathrm{H}_{2} \\
(J=0,2, \ldots)\end{array}$ & \multirow[b]{2}{*}{$\begin{array}{c}4158 \pm 3 \\
(34 \pm 2)\end{array}$} & $\begin{array}{c}Q_{1}(0) 4152 \\
(0.4)\end{array}$ & $\begin{array}{c}Q_{1}(0) 4154 \\
\quad(0.3)\end{array}$ & $\begin{array}{l}Q_{1}(0) 4161 \\
Q_{1}(2) 4143\end{array}$ \\
\hline & $\begin{array}{c}\text { ortho- }_{2} \\
(J=1,3, \ldots)\end{array}$ & & $\begin{array}{c}Q_{1}(1) 4143 \\
(0.4)\end{array}$ & $\begin{array}{c}Q_{1}(1) 4146 \\
(0.4)\end{array}$ & $\begin{array}{l}Q_{1}(1) 4155 \\
Q_{1}(3) 4125\end{array}$ \\
\hline \multirow[b]{2}{*}{$\mathrm{D}_{2}$} & $\begin{array}{c}\text { ortho- } \mathrm{D}_{2} \\
(J=0,2, \ldots)\end{array}$ & \multirow[b]{2}{*}{$\begin{array}{l}2990 \pm 2 \\
(20 \pm 1)\end{array}$} & $\begin{array}{c}Q_{1}(0) 2985 \\
(0.2)\end{array}$ & $\begin{array}{c}Q_{1}(0) 2988 \\
\quad(0.35)\end{array}$ & $\begin{array}{l}Q_{1}(0) 2994 \\
Q_{1}(2) 2987\end{array}$ \\
\hline & $\begin{array}{c}\text { para- } \mathrm{D}_{2} \\
(J=1,3, \ldots)\end{array}$ & & $\begin{array}{c}Q_{1}(1) 2982 \\
(0.2)\end{array}$ & $\begin{array}{c}Q_{1}(1) 2985 \\
(0.4)\end{array}$ & $\begin{array}{ll}Q_{1}(1) & 2991 \\
Q_{1}(3) & 2981\end{array}$ \\
\hline
\end{tabular}

"a Reference [13]. ${ }^{\mathrm{b}}$ Reference [12]. 


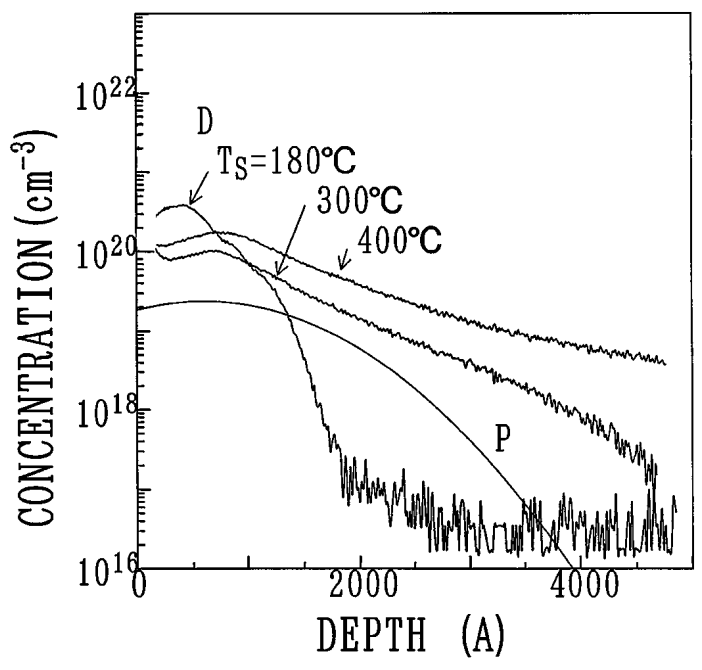

FIG. 3. Depth profiles of deuterium obtained by SIMS measurements for samples implanted at $5 \times 10^{14} \mathrm{P} / \mathrm{cm}^{2}$ and annealed at $900{ }^{\circ} \mathrm{C}$, followed by deuterium-atom treatment at substrate temperatures of 180,300 , and $400{ }^{\circ} \mathrm{C}$. Phosphorus profile was also measured and drawn schematically.

It was found that there were no significant changes in the vibrational Raman line at $4158 \mathrm{~cm}^{-1}$. Therefore, we conclude that the hydrogen molecules are located deeper sites in the bulk of the crystalline silicon, presumably at interstitial sites.

The hydrogen molecule $\mathrm{H}_{2}$ has two forms even in the solid state [15]; i.e., parahydrogen with antiparallel arrangement of the nuclear spins $(I=0)$ and orthohydrogen with $I=1$. The concentration ratio of the two kinds of hydrogen molecules is 1:3 in thermal equilibrium. It is expected that also in crystalline silicon the same case occurs at room temperature. However, only one broad line was observed for each of the samples HAT $-400{ }^{\circ} \mathrm{C}$ and DAT- $400{ }^{\circ} \mathrm{C}$. The broad lines $\left(\Delta \omega=34\right.$ and $\left.20 \mathrm{~cm}^{-1}\right)$ observed for $\mathrm{H}_{2}$ and $\mathrm{D}_{2}$ range between the frequency shifts corresponding to those from $4126 \mathrm{~cm}^{-1}$ for a higher rotational quantum number $J=3$ to $4161 \mathrm{~cm}^{-1}$ for $J=$ 0 , and those from $2980 \mathrm{~cm}^{-1}$ for a higher $J=3$ to $2994 \mathrm{~cm}^{-1}$ for $J=0$, respectively, by comparison with the results [12] for gas phases $\mathrm{H}_{2}$ and $\mathrm{D}_{2}$ shown in Table I. A rotational Raman line was also observed near $585 \mathrm{~cm}^{-1}$ in our samples which corresponds to the rotational line [ $\left.S_{0}(1): 586 \mathrm{~cm}^{-1}\right]$ of ortho- $\mathrm{H}_{2}[13]$.

Furthermore, we observed somewhat larger ESR linewidths for donor and/or conduction electrons at $4.2 \mathrm{~K}$ for the samples treated with deuterium atoms above $300{ }^{\circ} \mathrm{C}$ compared with the samples treated with hydrogen atoms. At lower temperatures, the para- $\mathrm{H}_{2}$ is dominant since its ground state is about $180 \mathrm{~K}$ lower in energy than that of the ortho- $\mathrm{H}_{2}$, while ortho- $\mathrm{D}_{2}$ is more stable than para- $\mathrm{D}_{2}$. The observed difference of the ESR linewidth may be due to the difference in the magnitude of the hyperfine interactions with donor and/or conduction electrons for the lowest-energy para- $\mathrm{H}_{2}(I=0)$ with $J=0$ and the lowest-energy ortho- $\mathrm{D}_{2}(I=0$ and 2$)$ with $J=0$.
In conclusion, we have provided direct evidence for the existence of hydrogen and deuterium molecules in crystalline silicon treated at $400{ }^{\circ} \mathrm{C}$ with atomic hydrogen and deuterium, respectively, by means of Raman scattering measurements. The Raman spectrum exhibits a vibrational line near $4158 \mathrm{~cm}^{-1}$, which is close to Raman lines associated with parahydrogen and orthohydrogen molecules in gas, liquid, and solid states, thereby unambiguously identifying the hydrogen molecules. To our knowledge, this is the first observation of hydrogen molecules in crystalline semiconductors or crystalline, inorganic materials.

We are grateful to C.A.J. Ammerlaan for helpful discussions about hydrogen molecules in $\mathrm{H}^{+}$-implanted silicon, and S. Yamasaki for giving information about hydrogen molecules in amorphous $\mathrm{Si}: \mathrm{H}$. This work is a part of a research contract from the NEDO.

[1] See, for example, Hydrogen in Semiconductors, edited by J.I. Pankove and N. M. Johnson, Semiconductor and Semimetals Vol. 34 (Academic Press, New York, 1991), and references therein.

[2] K. J. Chang and D. J. Chadi, Phys. Rev. Lett. 60, 1422 (1988).

[3] C. G. Van de Walle, Phys. Rev. B 49, 4579 (1994).

[4] M. Stutzmann, W. Beyer, L. Tapfer, and C.P. Herrero, Physica (Amsterdam) 170B, 240 (1991).

[5] P. Stallinga, T. Gregorkiewicz, C. A. J. Ammerlaan, and Yu. V. Gorelkinskii, Phys. Rev. Lett. 71, 117 (1993).

[6] K. L. Brower, S. M. Myers, A. H. Edwards, N. M. Johnson, C. G. Van de Walle, and E. H. Poindexter, Phys. Rev. Lett. 73, 1456 (1994).

[7] K. J. Chang and D. J. Chadi, Phys. Rev. Lett. 62, 937 (1989); D. J. Chadi and C. H. Park, Phys. Rev. B 52, 8877 (1995).

[8] J. D. Holbech, B. B. Nielsen, R. Jones, P. Sitch, and S. Oberg, Phys. Rev. Lett. 71, 875 (1993).

[9] J. B. Boyce and M. Stutzmann, Phys. Rev. Lett. 54, 562 (1985).

[10] Y.J. Chabal and C. K. N. Patel, Phys. Rev. Lett. 53, 210 (1984).

[11] M. Kitajima, K. Aoki, and M. Okada, J. Nucl. Mater. 149, 269 (1987).

[12] B. P. Stoicheff, Can. J. Phys. 35, 730 (1957).

[13] S. S. Bhatnagar, E. J. Allin, and H. L. Welsh, Can. J. Phys. 40, 9 (1962).

[14] J. Kikuchi, S. Fujimura, M. Suzuki, and H. Yano, Jpn. J. Appl. Phys. 32, 3120 (1993).

[15] N. Fukata, S. Fujimura, and K. Murakami, Mater. Sci. Forum 196-201, 873 (1995); N. Fukata, S. Sasaki, S. Fujimura, H. Haneda, and K. Murakami, Jpn. J. Appl. Phys. (to be published).

[16] N. M. Johnson, F. A. Ponce, R. A. Street, and R. J. Nemanich, Phys. Rev. B 35, 4166 (1987).

[17] K. Murakami, H. Suhara, S. Fujita, and K. Masuda, Phys. Rev. B 44, 3409 (1991).

[18] H. Richter, Z. P. Wang, and L. Ley, Solid State Commun. 39, 625 (1981). 